Martijn R. Meijerink

Jan Hein T. M. van Waesberghe

Lineke van der Weide

Petrousjka van den Tol

Sybren Meijer

Cornelis van Kuijk

\section{Total-liver-volume perfusion CT using 3-D image fusion to improve detection and characterization of liver metastases}

Received: 3 December 2007

Revised: 26 February 2008

Accepted: 8 March 2008

Published online: 20 May 2008

(C) The Author(s) 2008

M. R. Meijerink $(\bowtie)$

J. H. T. M. van Waesberghe .

C. van Kuijk

Department of Radiology, Vrije

Universiteit Medical Center (VUMC),

De Boelelaan 1117,

1081HV Amsterdam, The Netherlands

e-mail: mr.meijerink@vumc.nl

Tel.: +31-20-4440362

Fax: +31-20-4440399

L. van der Weide

Master of Oncology Program,

Vrije Universiteit Medical Center,

Amsterdam, The Netherlands

P. van den Tol $\cdot$ S. Meijer

Department of Surgical Oncology,

Vrije Universiteit Medical Center,

Amsterdam, The Netherlands

\begin{abstract}
The purpose of this study was to evaluate the feasibility of a totalliver-volume perfusion CT (CTP) technique for the detection and characterization of liver metastases. Twenty patients underwent helical CT of the total liver volume before and 11 times after intravenous contrast-material injection. To decrease distortion artifacts, all phases were co-registered using 3-D image fusion before creating blood-flow maps. Lesion-based sensitivity and specificity for liver metastases of first the conventional four phases (unenhanced, arterial, portal venous, and equilibrium) and later all 12 phases including blood-flow maps were determined as compared to intraoperative ultrasound and surgical exploration. Arterial and portal venous perfusion was calculated for normalappearing and metastatic liver tissue.
\end{abstract}

Total-liver-volume perfusion values were comparable to studies using single-level CTP. Compared to fourphase CT, total -liver-volume CTP increased sensitivity to 89.2 from $78.4 \%(P=0.046)$ and specificity to 82.6 from $78.3 \%(P=0.074)$. Total liver-volume CTP is a noninvasive, quantitative, and feasible technique. Preliminary results suggest an improved detection of liver metastases for CTP compared to four-phase CT.

Keywords Perfusion - Computed tomography $(\mathrm{CT}) \cdot$ Liver metastasis

\section{Introduction}

Until recently the only potentially curative strategy for patients with colorectal liver metastases was surgical resection. During the last few years, several alternative local ablative therapies, such as radiofrequency ablation, have also been suggested to improve survival $[1,2]$. The results of these local forms of therapy strongly depend on the accuracy of pre- and intraoperative imaging. Any technique that increases sensitivity and specificity for the detection of liver metastases and that improves the evaluation of local tumor extent will therefore probably not only improve survival but may also prevent patients from receiving unnecessary invasive forms of surgery.

In the preoperative assessment of liver tumors, singlephase computed tomography (CT) is often insufficient to characterize focal liver lesions due to the variable enhancement patterns of liver tumors compared to the relatively constant enhancement pattern of normal liver parenchyma. Moreover single-phase $\mathrm{CT}$ is inferior to multiphase CT in the detection of focal liver lesions [3-5]. Therefore, multiphase CT has been widely accepted as the imaging modality of first choice either for the workup of focal liver lesions or as the primary imaging modality when suspicion of liver malignancy is high. Adding more phases may further improve detection and characterization of focal liver lesions.

Since the early 1990 s, a single-level, dynamic, contrastenhanced perfusion CT (CTP) technique that allows for the quantification and mapping of hepatic tumor blood-flow parameters such as tissue perfusion has been examined by a relatively small group of investigators [6-19]. CT 
measurements of hepatic perfusion amongst patients with colon cancer have been shown to correlate with patient survival in two small groups of patients $[10,11]$ and to provide risk stratification possibly even superior to the Dukes' classification $[18,19]$. Furthermore, the presence of a focal arterial hyperdense blush and a general increase in global hepatic artery tissue perfusion on CTP may indicate the presence of occult micrometastasis $[8$, 9]. CTP has also been successful in demonstrating a significant decrease of $40-44 \%$ in tumor blood perfusion after antiangiogenic therapy, and therefore CTP may serve as a biomarker for tumor response [20].

Two major limitations, however, have hampered the use of CTP. Because of the necessity of a high temporal resolution to produce reliable enhancement curves for every voxel within a certain volume, older CT machines were simply not fast enough to acquire repeated examinations of the entire liver every few seconds. Secondly, heterogeneity in either inspiratory or expiratory volumes between two successive breathing in and out commands have previously produced severe motion and distortion artifacts of the liver and have so severely degraded the quality of enhancement curves that reliable calculations of perfusion parameters were impossible.

The purpose of this study was to evaluate the feasibility of a total-liver-volume CTP technique for the detection and characterization of liver metastases. The authors describe their early experience with this ultrafast total-liver-volume contrast-enhanced perfusion $\mathrm{CT}$, in which an automated 3$\mathrm{D}$ image fusion technique was applied to decrease motion and distortion artifacts induced by differences in inspiratory volume.

\section{Materials and methods}

\section{Patient selection}

Between July 2006 and March 2007, 20 consecutive patients (14 male; 6 female) suitable for surgical resection and/or radiofrequency ablation of liver metastases were selected for this study. The mean age was 64 years (range, 48-80 years). Nineteen patients had liver metastases from primary colorectal carcinoma, and one patient had liver metastases from an adenocarcinoma of unknown primary origin. Additional selection criteria were a WHO performance status of 0-2 [21], a Karnofsky performance status (KPS) $\geq 80 \%$, a life expectancy of more than 3 months as well as adequate hematological, renal and hepatic function, measured as serum hemoglobin level, platelet and white blood cell count, creatinin and bilirubin within the upper limit of normal. Patients with known contrast-medium reactions, patients unable to hold their breath for a sufficient period of time, and patients unable to obey breath-hold commands were excluded. The nutritional status during CT examination was normal in all patients (no fasting period). All patients underwent CT no more than $24 \mathrm{~h}$ before surgery; the entire liver was examined by both visual and palpatory inspection and intraoperative ultrasound (IOUS). The study was approved by the institutional ethical and scientific review board, and all patients gave written informed consent.

\section{Imaging protocol}

Dynamic CTP measurements were obtained with a 64-slice multi-detector CT system (Somatom Sensation, Siemens, Erlangen, Germany). A 12-phase helical CT examination of the total liver volume (collimation $0.6 \mathrm{~mm}$; rotation time $0.33 \mathrm{~s}$; pitch 0.75 ) was acquired before and 11 times after rapid intravenous injection $(6 \mathrm{ml} / \mathrm{s})$ of $100 \mathrm{ml}$ low-osmolar nonionic contrast agent with an iodine concentration of $300 \mathrm{mg} / \mathrm{ml}$ (Ultravist-300 Iopromide; Schering, Berlin, Germany) and $20 \mathrm{ml}$ saline chasing bolus into the left antecubital vein, using an injection pump through an 18 -g needle.

Bolus tracking (threshold $+100 \mathrm{HU}$ ) was used to start the CT data acquisition, placing a region of interest (ROI) over the right atrium. With a minimum interscan delay of 3-4 s in between two series, the first four series were obtained in one single breath-hold at maximum inspiration. After these first series, one breath-out and breath-in command (lasting 8 s) was given before adding two series in one single breath-hold at maximum inspiration. This was repeated until a total of 11 series were acquired. The 10th series (considered as the portal venous phase series) was obtained during a single breath hold in which the entire (upper and lower) abdomen was examined at conventional tube voltage and current (120 kV and maximum $180 \mathrm{mAs}$ with dose modulation). In all other series, a fixed lower tube current was used to reduce radiation exposure $(120 \mathrm{kV}$ and $80 \mathrm{mAs})$. The 12th and final series was performed at equilibrium after approximately $300 \mathrm{~s}$. The timetable of this protocol is displayed in Fig. 1. All series were reconstructed in both 5-mm contiguous axial slices and thin overlapping axial slices (slice width $1.5 \mathrm{~mm}$; reconstruction increment $1.0 \mathrm{~mm}$ ) for optimum visualization in the multiplanar reconstruction mode of the 3-D fusion program.

\section{Radiation exposure}

The effective dose, expressed according to the International Commission on Radiological Protection recommendations measured with commercially available software was approximately $24.0 \mathrm{mSv}(11 \times 1.5 \mathrm{mSv}+1 \times 7.5 \mathrm{mSv})$ for the dynamic CTP protocol [22-24]. For comparison, using the same scan range, the effective dose for only the conventional four phases used in our protocol is approximately $12.0 \mathrm{mSv}(3 \times 1.5 \mathrm{mSv}+1 \times 7.5 \mathrm{mSv})$. However, for 


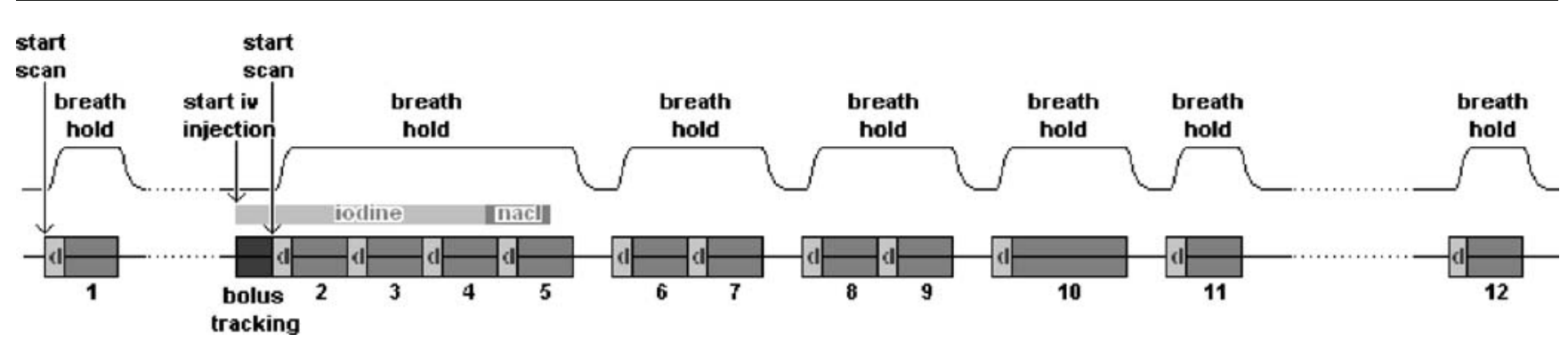

Fig. 1 Timetable of the computed tomography perfusion protocol. All series were acquired during breath-hold at maximum inspiration, 1 series before and 11 series after the rapid administration of intravenous contrast material. The delay $(d)$ between two acquisitions was $3 \mathrm{~s}$

a conventional-dose four-phase CT this would be approximately $20.7 \mathrm{mSv}(3 \times 4.4 \mathrm{mSv}+1 \times 7.5 \mathrm{mSv})$.

\section{Postprocessing}

Initially the four-phase CT images [unenhanced and at $30 \mathrm{~s}$ (5th or arterial phase), $70 \mathrm{~s}$ (10th or portal venous phase) and $300 \mathrm{~s}$ (12th phase or equilibrium) after start of injection] were extracted from the 12-phase CTP scan. An experienced radiologist (J.H.v.W) unaware of the results of the CTP evaluated the four phases and scored all visible liver lesions as appearing either malignant or benign.

Image fusion was performed with a commercially available 3-D image fusion program (Vinci 2.36.0, Max
Planck Institute for Neurological Research, Cologne). The 12 series were fused by a combination of automated and manual image shifting using colored transparent image overlay methods. The 10th (portal venous phase) series was chosen as reference to which all other series were registered in 5-mm contiguous axial slices. The time necessary for the entire registration process of a single examination was approximately $20-40 \mathrm{~min}$. To assess the effect of image fusion, time-density curves of subdiaphragmatic regions of interest (ROIs), which are most vulnerable to artifacts induced by differences in inspiratory volume, were drawn on the most superior slice below the diaphragm on the portal venous phase and subsequently copied to all other phases before and after image registration (Fig. 2). Time-density curves were considered
A

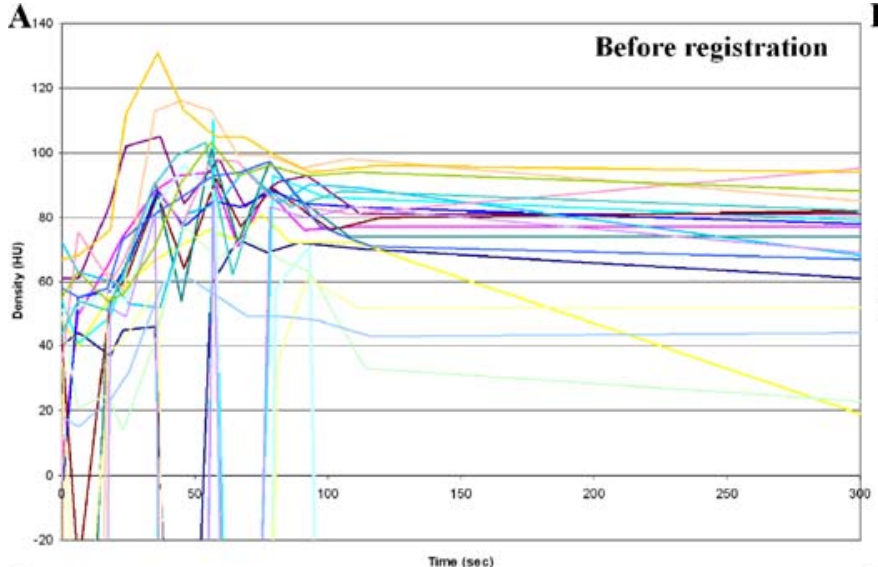

C

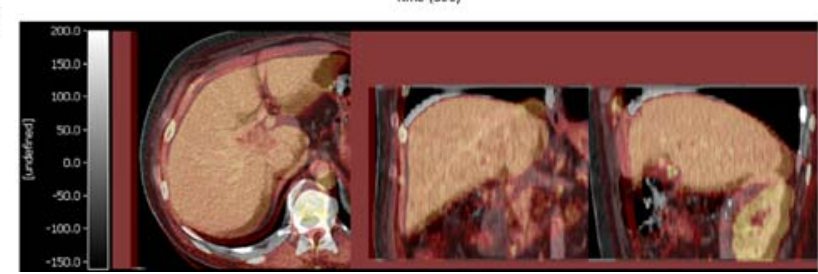

Fig. 2 Graphs showing the time-density curves of ROIs placed in the most superior slice below the diaphragm of the portal venous reference phase, which is highly vulnerable to artefacts induced by differences of inspiratory volume. The curves were subsequently copied to all other phases, before $(\mathbf{a}, \mathbf{c})$ and after $(\mathbf{b}, \mathbf{d}) 3-\mathrm{D}$ image

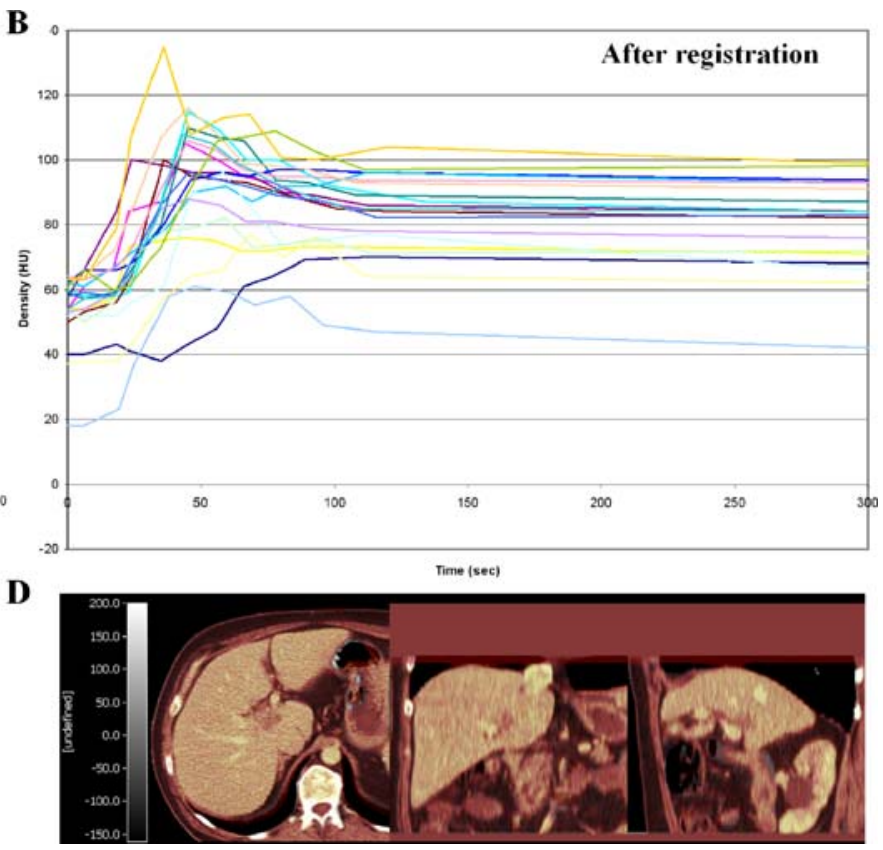

fusion and co-registration. The improved shape of the timeenhancement curves after image fusion will decrease distortion artifacts on the blood-flow maps and lead to more reliable perfusion values 
unacceptable for calculation of perfusion and creation of blood-flow maps if at least one time point had a density of $>10 \mathrm{HU}$ below the precontrasted liver parenchyma or if a decrease of $>30 \mathrm{HU}$ between two time points was present, most likely representing the inclusion of lung parenchyma or fatty extrahepatic tissue within the ROI.

For the quantification of tissue perfusion and for the creation of blood-flow maps, the software program Basama Perfusion 3.0.7.1 (Kanazawa, Ishikawa, Japan) was used [16]. ROIs were placed within normal-appearing liver parenchyma, within the hypodense lesions as determined on the portal venous phase images and within a rim region of approximately $1 \mathrm{~cm}$ surrounding these hypodense lesions. The program estimates tissue perfusion as the maximum slope of the tumor time-density curve divided by the peak arterial enhancement. The mathematical technique has previously been fully described elsewhere $[6,7,25]$. Due to the dual blood supply of the liver, hepatic tissue perfusion is divided into hepatic artery and portal venous perfusion as the maximum slope of the tumor time-density curve before versus after the splenic peak enhancement divided by, respectively, the peak aortic and portal enhancement. For an example of the total volume CTP blood-flow maps, see Fig. 3. The time necessary for quantifying perfusion and creation of total-liver-volume blood-flow maps of a single examination was approximately $20-40 \mathrm{~min}$.

After at least 1 month to reduce recall bias, the same experienced radiologist (J.H.v.W.) evaluated all 12 phases including the hepatic artery and portal venous blood-flow maps and again scored all visible lesions as appearing either malignant or benign. Large lesions $(>2 \mathrm{~cm})$ were considered to appear malignant on blood-flow maps if a low central and high peripheral arterial perfusion was found in combination with a low portal venous perfusion compared to adjacent normal-appearing liver tissue. Small lesions $(<2 \mathrm{~cm})$ were also considered to appear malignant if the entire lesion presented with high arterial perfusion.

Previous porcine studies have shown that as cardiac output decreases, the times to the arrival of the contrastmedium bolus in the aorta and to peak aortic and hepatic enhancement increase. Furthermore, reduction in cardiac output results in a substantial increase in peak aortic enhancement but not in peak hepatic enhancement [26]. Therefore, although somewhat arbitrary, to estimate the influence of cardiac output on the perfusion parameters, we used the time-to-peak and peak-enhancement values as surrogate markers for cardiac output. Both arterial and portal venous time-to-peak measurements and peakenhancement values were compared to the perfusion values.
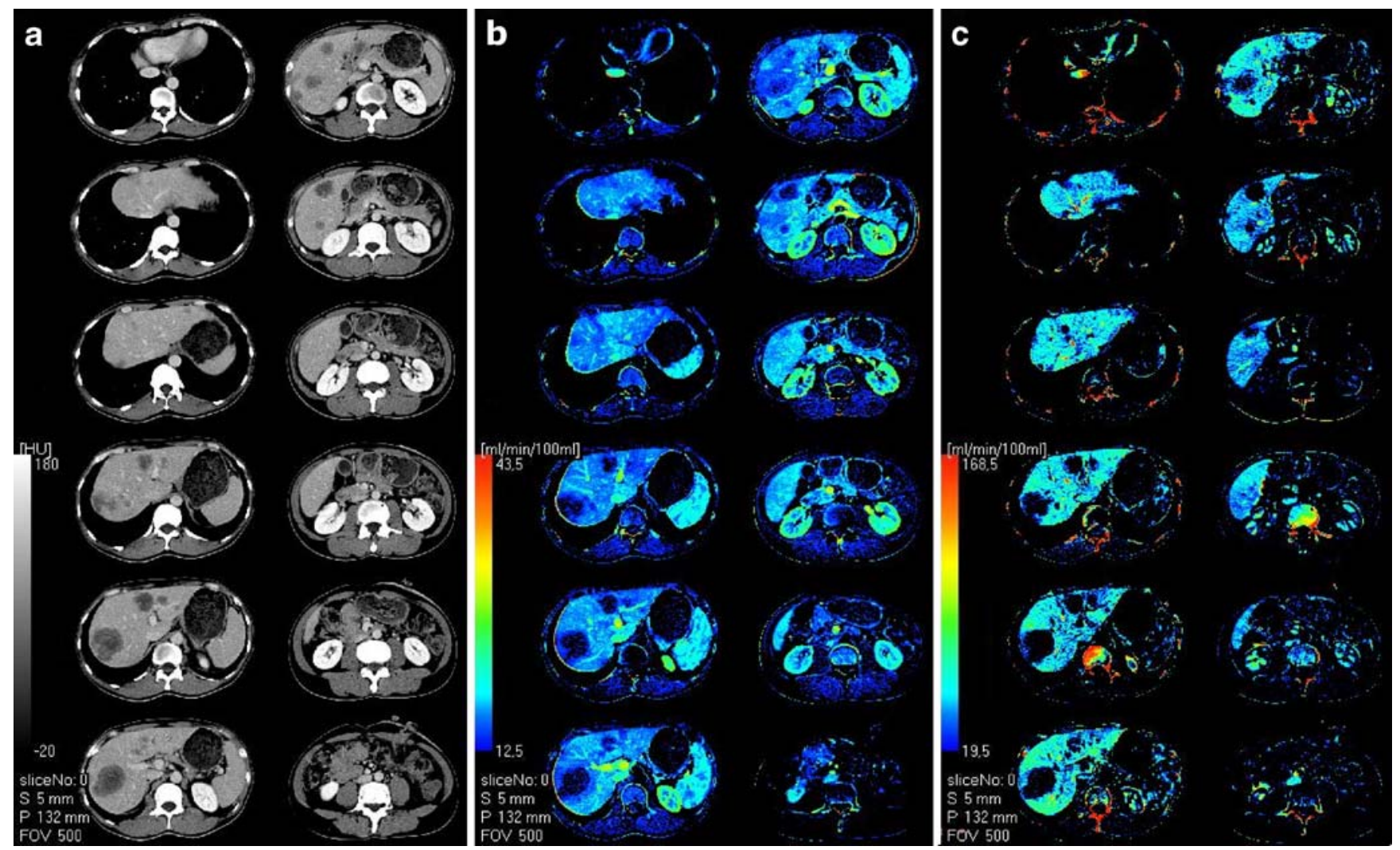

Fig. 3 Total-volume CTP showing a selection of the portal venous phase images (a) and the hepatic artery (b) and portal vein (c) bloodflow maps in a patient with multiple colorectal liver metastases 


\section{Statistical analysis}

To establish lesion-based sensitivity and specificity both four-phase CT and CTP techniques were compared to a gold standard of surgical exploration and IOUS. Pearson's product moment correlation coefficient was used to determine the correlation between cardiac output estimations and the perfusion values [correlation coefficients $(r)$ were considered as follows: $<0.2$ negligible, $0.2-0.4$ weak, $0.4-0.7$ moderate, $0.7-0.9$ strong, and $>0.9$ very strong correlation].

\section{Results}

In all patients, the CT protocol, the rapid injection of contrast medium, and the breath-hold commands were well tolerated. No patients had to be excluded.

\section{Influence of image fusion}

In $14 / 20$ cases, before image fusion and registration, the time-density curves of subdiaphragmatic ROIs were considered unacceptable to calculate perfusion or to create blood-flow maps due to the presence of lung parenchyma or fatty extrahepatic tissue within the ROI at at least one time point. After image fusion and registration all 20/20 time-density curves could be considered acceptable; no lung parenchyma or fatty extrahepatic tissue was present within the ROIs (Fig. 2).

\section{Influence of cardiac output}

The peak enhancement in the aorta on average was $368 \pm$ $61 \mathrm{HU}$ (range 259-471 HU) and the peak enhancement in the portal vein was $188 \pm 32 \mathrm{HU}$ (range 139-243 HU). The average time-to-peak was $20.5 \pm 5.8 \mathrm{~s}$ (range 8-36 s) for the aorta and $37.4 \pm 13.0 \mathrm{~s}$ (range 18-77 s) for the portal vein. A shorter time-to-peak showed moderate to strong correlation with higher peak enhancement ( $r=0.62$ for the aorta and $r=$ 0.71 for the portal vein). The aortic $(r=0.39)$ and portal vein $(r=0.17)$ times-to-peak and the peak aortic $(r=0.37)$ and peak portal vein $(r=0.36)$ enhancement all showed weak or negligible correlations with perfusion values for normal liver tissue, although a trend towards higher perfusion in patients with higher peak enhancements and lower time-to-peak values was present.

\section{Evaluation of blood-flow maps}

Almost all lesions were of considerably larger volume when evaluated on the portal venous blood-flow maps compared to the conventional portal venous phase images before reconstruction. This volume mismatch resembles a rim region that surrounds the hypodense and mainly necrotic lesions visible on conventional $\mathrm{CT}$, and that has a very high hepatic artery flow (Fig. 4) of $32.1 \mathrm{ml} \mathrm{min}$ $100 \mathrm{~g}^{-1}$ on average. In calculating the portal venous perfusion of the voxels within this region, the larger postpeak dip of the hepatic artery perfusion curve superimposes on the post-splenic-peak portal venous enhancement curve. This may cause a decrease or even subzero post-splenicpeak maximum slope, which would falsely lead to a diminished or even negative portal venous flow. Portal venous perfusion in these areas is therefore probably underestimated and highly unreliable as has been previously described [20].

\section{Detection and characterization of focal liver lesions}

For the determination of sensitivity and specificity in the detection of liver metastases, one patient had to be excluded due to the preoperative presence of severely progressive disease; more than 20 malignant-appearing
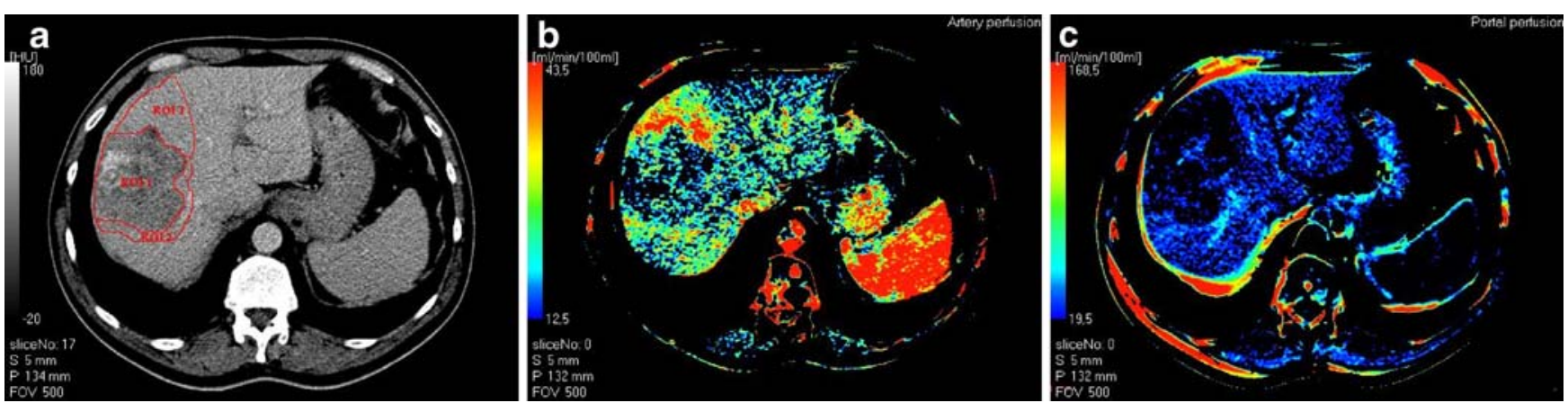

Fig. 4 Total-volume CTP showing the portal venous phase images (a) and the hepatic artery (b) and portal venous (c) blood-flow maps in a patient with a large colorectal liver metastasis. The mainly necrotic metastasis (ROI1) is surrounded by a rim region with high hepatic artery flow (ROI2) and bordered ventrally by a wedgeshaped region with very high hepatic artery flow and absent or immeasurable portal venous blood flow (ROI3) 
liver lesions were found (all visible on both the four-phase CT images and the CTP images) making him unsuitable for further surgical exploration and IOUS. In two other patients, the only visible liver lesions appeared to be benign, representing either simple cysts or hemangiomas. Since in both cases, no surgical exploration or IOUS was performed, the absence of PET avidity, the typical aspect on targeted US examinations, and the absence of change or growth after 3 months were used as alternative gold standard. Based on the four-phase CT images alone a total of 34 malignant-appearing lesions (29 true positives and 5 false positives: 3 simple hepatic cysts and 2 hemangiomas) and 13 benign-appearing lesions were found (all true negatives: 4 simple hepatic cysts, 1 hemangioma, 5 fully cystic, well-margined post-RFA lesions, and 3 benign focal perfusion alterations).

All 29 true-positive colorectal liver metastases (CRLM) could be visualized on the portal venous phase images, however the unenhanced and hepatic artery phase helped characterize at least 5 small CRLM. To correctly diagnose the hemangioma as a true benign "fill-in" lesion, the equilibrium phase was indispensable. By adding the additional CTP phases and the hepatic artery and portal venous blood-flow maps to these conventional CT images, a total of 37 malignant-appearing lesions (33 true positives and 4 false positives: all 4 representing benign superficial wedge-shaped focal perfusion alterations) and 16 benignappearing lesions were found (all true negatives: 7 simple hepatic cysts, 3 hemangiomas, 5 post-RFA lesions, and 1 benign focal perfusion alteration). In the gold standard group, a total of 37 liver metastases and 18 benign liver lesions ( 7 simple hepatic cysts, 6 hemangiomas, and 5 postRFA lesions) were detected; 5 lesions detected on CTP represented benign focal perfusion alterations. For a summary of the results see Table 1 .

Two examples of liver metastases undetected with fourphase CT and detected with CTP are given in Fig. 5 and
Fig. 6. For the four-phase CT versus the CTP techniques, we found a sensitivity of $78.4 \%$ versus $89.2 \%(P=0.046)$, respectively, and a specificity of $78.3 \%$ versus $82.6 \%$ $(P=0.078)$. The positive predictive value of malignantappearing lesions was $85.3 \%$ versus $89.2 \%$ for four-phase CT and CTP, respectively; the negative predictive value of benign-appearing lesions was $100.0 \%$ for both four-phase CT and CTP. Compared to the four-phase CT, four additional liver metastases (in three patients) were detected with CTP and eight (in four patients including the previously mentioned three patients) with surgical exploration and IOUS. The four additionally detected lesions found with CTP all represented small lesions $(<2.0 \mathrm{~cm})$ first visualized on the blood-flow maps, although retrospectively three out of four lesions were also vaguely visible on one of the 12-phases. Histology confirmed the metastatic nature in three of four additionally detected CRLM. In the fourth additionally detected lesion no histology was obtained due to progressions of disease elsewhere in the liver (Fig. 5).

All liver metastases undetected with either four-phase CT or CTP represented very small lesions $(<1.0 \mathrm{~cm})$. In three out of four of these patients the treatment strategy changed to no further local therapy, due to the technical inability to resect and/or ablate all lesions. Since all four falsely positive superficial wedge-shaped focal perfusion alterations (in two patients) appeared small and technically resectable on perfusion CT, retrospectively, the CTP results would not have changed the decision to perform surgery.

\section{Quantification}

Average perfusion of the normal-appearing liver parenchyma was $18.7 \pm 7.1 \mathrm{ml} \mathrm{min}^{-1} 100 \mathrm{~g}^{-1}$ for the hepatic artery (range $8.5-33.2 \mathrm{ml} \mathrm{min}^{-1} 100 \mathrm{~g}^{-1}$ ) and $57.2 \pm 20.6 \mathrm{ml}$ $\min ^{-1} 100 \mathrm{~g}^{-1}$ for the portal vein (range $23.3-108.3 \mathrm{ml}$

Table 1 Contingency tables with results for four-phase CT and CTP techniques

\begin{tabular}{|c|c|c|c|}
\hline & Malignant & Benign & Total \\
\hline \multicolumn{4}{|l|}{ Four-phase CT compared to the gold standard ${ }^{\mathrm{a}}$} \\
\hline Malignant-appearing lesions & $29(48 \%)$ & $5(8 \%)$ & $34(57 \%)$ \\
\hline Benign-appearing lesions & $0(0 \%)$ & $13(22 \%)$ & $13(22 \%)$ \\
\hline Lesions undetected with four-phase CT but detected with gold standard & $8(13 \%)$ & $5(8 \%)$ & $13(22 \%)$ \\
\hline Total & $37(62 \%)$ & $23(38 \%)$ & $60(100 \%)$ \\
\hline \multicolumn{4}{|l|}{ CTP compared to the gold standard ${ }^{\mathrm{a}}$} \\
\hline Malignant-appearing lesions & $33(55 \%)$ & $4(7 \%)$ & $3762 \%)$ \\
\hline Benign-appearing lesions & $0(0 \%)$ & $16(27 \%)$ & $16(27 \%)$ \\
\hline Lesions undetected with CTP but detected with gold standard & $4(7 \%)$ & $3(5 \%)$ & $7(12 \%)$ \\
\hline Total & $37(62 \%)$ & 23 38\%) & $60(100 \%)$ \\
\hline
\end{tabular}

${ }^{\mathrm{a} C o m b i n a t i o n}$ of surgical exploration and IOUS (gold standard) 

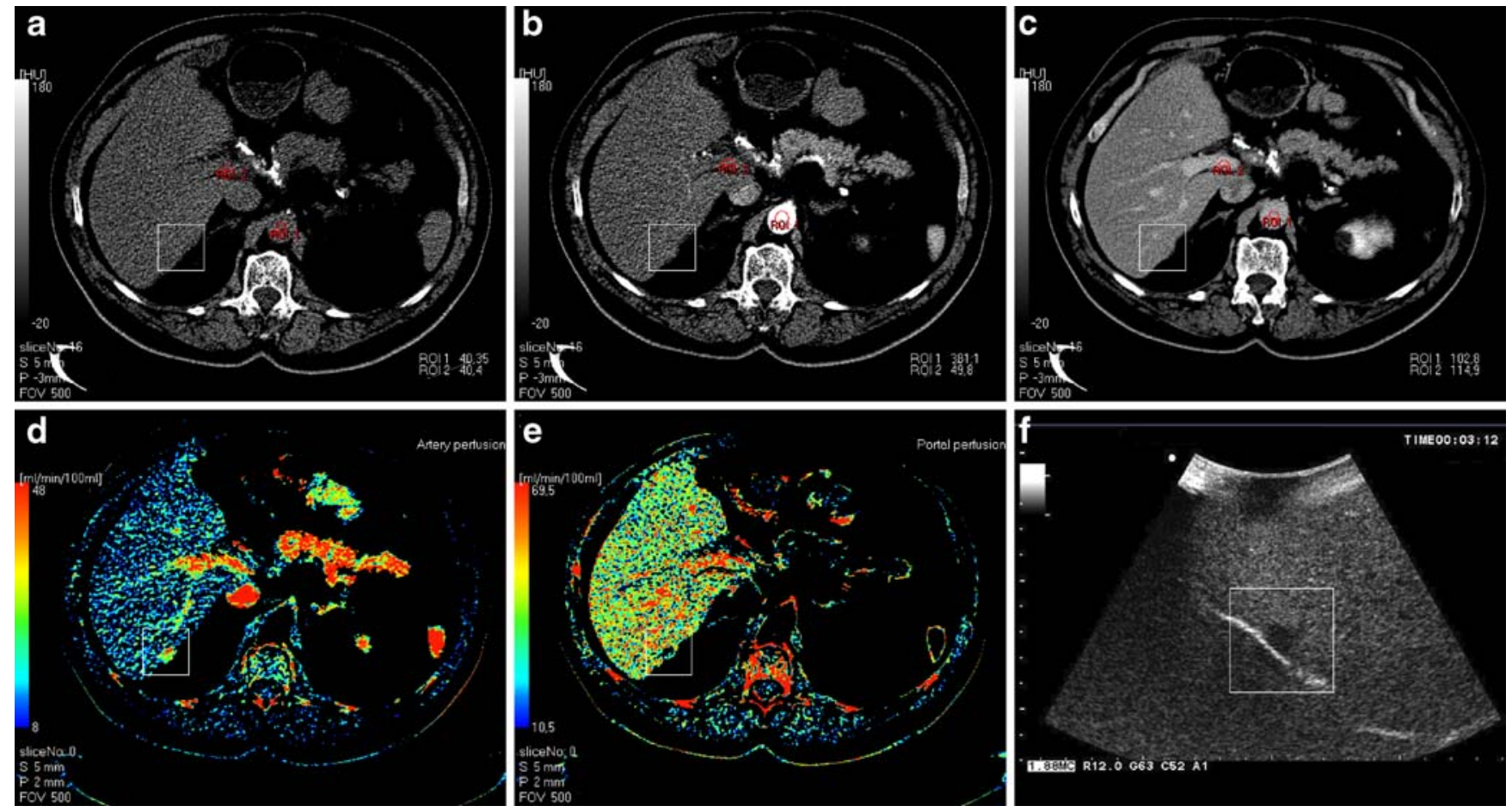

Fig. 5 Example of a small liver metastasis (white boxes) undetected with four-phase CT [unenhanced (a); arterial phase (b); portal venous phase (c)] and detected on the CTP blood-flow maps [arterial (d) and portal vein (e)]. Surgical inspection and palpation of the

local liver surface and IOUS (f) clearly confirmed the metastatic nature of the lesion, although no histology was obtained due to the progression of disease elsewhere in the liver

$\min ^{-1} 100 \mathrm{~g}^{-1}$ ); hepatic artery contribution to the hepatic blood flow (hepatic perfusion index or HPI) of normalappearing liver parenchyma was $26 \pm 11 \%$ (range $13-57 \%$ ). Average perfusion of the hypodense liver metastases was $8.2 \pm 5.3 \mathrm{ml} \mathrm{min}^{-1} 100 \mathrm{~g}^{-1}$ for the hepatic artery (range 0 $16.9 \mathrm{ml} \mathrm{min}^{-1} 100 \mathrm{~g}^{-1}$ ) and $17.0 \pm 16.5 \mathrm{ml} \mathrm{min}^{-1} 100 \mathrm{~g}^{-1}$ for the portal vein (range $0-63.3 \mathrm{ml} \mathrm{min}^{-1} 100 \mathrm{~g}^{-1}$ ); HPI was $44 \pm 32 \%$ (range $0-100 \%$ ). Average perfusion of the rim regions surrounding these hypodense lesions was $32.1 \pm$ $12.9 \mathrm{ml} \mathrm{min}^{-1} 100 \mathrm{~g}^{-1}$ for the hepatic artery (range 12.6$56.4 \mathrm{ml} \mathrm{min}^{-1} 100 \mathrm{~g}^{-1}$ ) and $35.5 \pm 24.8 \mathrm{ml} \mathrm{min}^{-1} 100 \mathrm{~g}^{-1}$ for the portal vein (range $0-89.4 \mathrm{ml} \mathrm{min}^{-1} 100 \mathrm{~g}^{-1}$ ); HPI was $51 \pm 23 \%$ (range $23-100 \%$ ).

\section{Discussion}

The preliminary results of this study suggest an improved detection rate of colorectal liver metastases for the totalvolume CTP-based technique with 3-D image fusion. The technique might also improve differentiation between benign and malignant liver lesions, although distinction between superficial wedge-shaped areas of nonmetastatic altered liver tissue perfusion and the hyperdense blush surrounding occult liver metastases seems impossible. Therefore, the number of pre-procedurally detected liver metastases may be somewhat overestimated compared to

four-phase CT. As a result, the mere presence of these superficial wedge-shaped focal perfusion alterations on CTP-derived blood-flow maps cannot exclude patients from receiving surgery. For this reason the number of patients in whom CTP will prevent unnecessary surgery will probably be limited. Nevertheless, the presence of these focal perfusion alterations can guide surgical inspection and target IOUS to the presence of small and previously occult liver metastases.

One important limitation of the study is the lower tube current of $80 \mathrm{mAs}$ used for the unenhanced, arterial, and equilibrium phases to reduce radiation exposure. The most important phase for detection of colorectal liver metastases, namely the portal venous phase, was obtained with conventional higher tube currents. Although in theory these lower tube currents could have negatively influenced the results of the four-phase CT, in all three patients where additional liver metastases were found with CTP, a conventional-dose four-phase CT had been performed no more than 6 weeks before surgery. The conventional-dose four-phase CT also did not reveal the lesions (both in the radiology report and by retrospective blinded evaluation).

Both the ratio of hepatic artery over portal venous perfusion and the arterial and portal venous perfusion values of normal-appearing liver tissue are in accordance with other studies using the direct maximum slope method, where arterial versus portal venous perfusion ranged from 

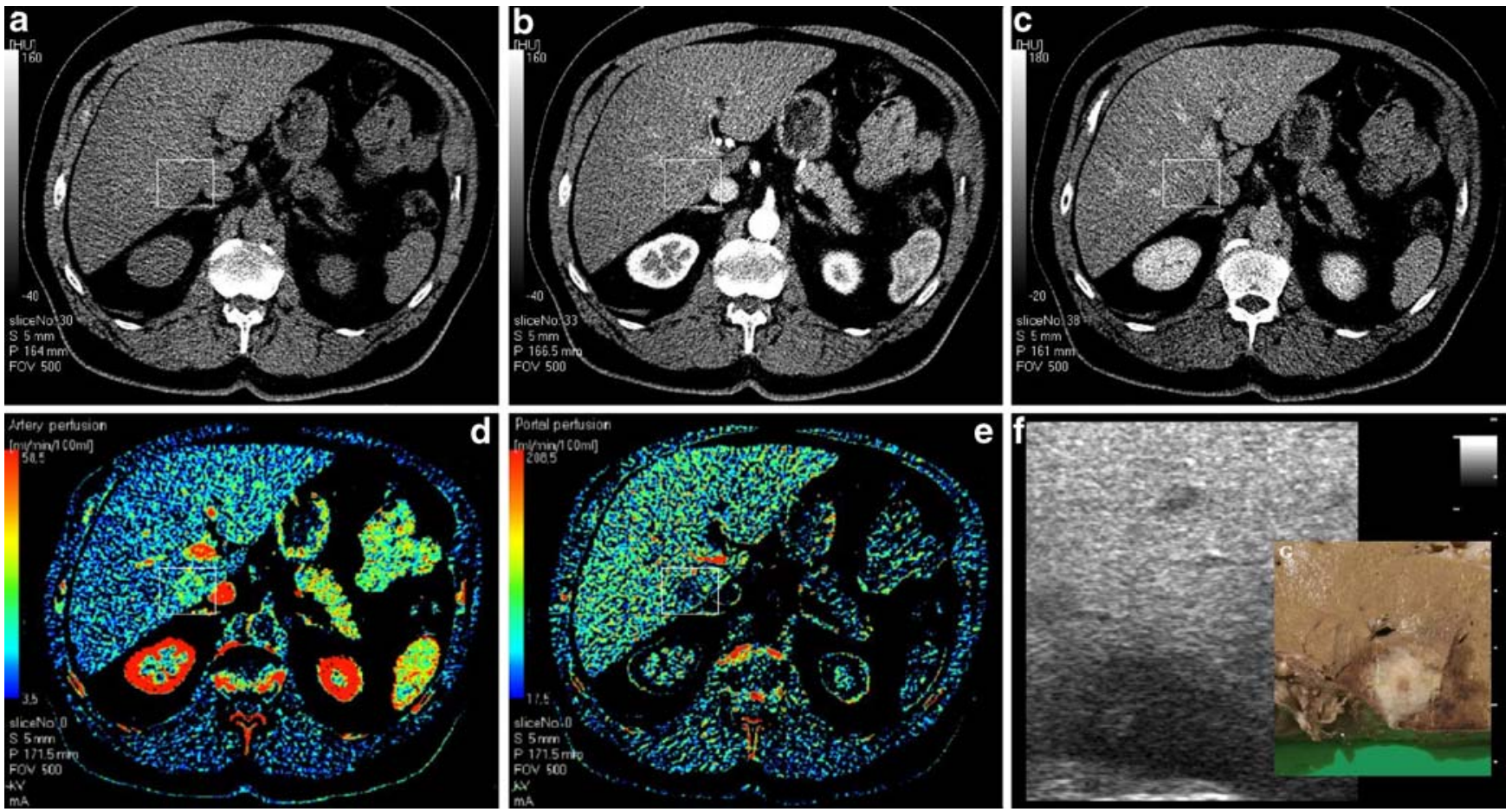

Fig. 6 Another example of liver metastasis (white boxes) undetected with four-phase CT [unenhanced (a); arterial phase (b); portal venous phase (c)] and detected on the CTP blood-flow maps [arterial (d) and portal venous (e)]. Retrospectively a vague heterogeneous lesion can be identified on the arterial and portal venous phase images. At surgery, the lesion appeared irresectable

17 to $30 \mathrm{ml} \mathrm{min}^{-1} 100 \mathrm{~g}^{-1}$ versus 34 to $106 \mathrm{ml} \mathrm{min}^{-1} 100 \mathrm{~g}^{-1}$ $[7,8,15,17,27]$.

The higher ratio of hepatic artery over portal venous flow in the center of malignant lesions as well as in rim regions surrounding nearly all colorectal liver metastases is fully comparable to results from other studies. The hypothetical mechanisms that lead to these observations are difficult and most likely heterogeneous and multifactorial. First, for reasons that are not presently understood, the proliferation of blood vessels that are dependent on vascular endothelial growth factor in colorectal liver metastases (angiogenesis) favors the recruitment of hepatic artery branches over portal veins [28]. These blood-flow alterations may be paralleled within a certain region surrounding the tumor due to the local spread of tumoral cytokines. Secondly, mechanical tumor compression and/ or peritumoral vessel lumen-narrowing secondary to fixed leukocyte adherence will result in reduced portal flow [29]. Although under debate, decreased portal supply in the compressed hepatic parenchyma has been suggested to evoke a compensatory increase in arterial blood supply [30]. Hepatic artery perfusion in these areas is probably overestimated due to the decreased dilution within the hepatic sinusoids of the highly dense contrasted arterial blood with the not-yet-contrasted portal venous blood. The combination of an increased arterial blood supply and but suitable for RFA (f). Three days after surgery and RFA, the 80year-old male patient died of an autopsy-proven massive myocardial infarction. At histology, the lesion was confirmed as a post-RFA (needle tract and ablation zone clearly visible) colorectal liver metastasis (g)

compression of hepatic veins might even produce local reversion of flow resulting in draining portal veins [10]. Thirdly and fourthly, rim enhancement has been described to be caused by the drainage of blood flow from the tumor to the surrounding hepatic parenchyma in hypervascular metastases or by increased arterioportal shunts surrounding the tumor in hypovascular metastases [31]. Finally, perilesional enhancement has been reported to correlate with histopathologic hepatic parenchymal changes, such as inflammatory cell infiltration and hyperemia and the presence of a desmoplastic reaction [32].

Since true separation of the hepatic artery and portal venous tissue time-enhancement curves is impossible using CTP, the arterial and portal fractions of the liver perfusion can only be estimated. Mathematical models that do so, therefore, all have certain limitations. The direct maximum slope method uses the temporal interval between the presplenic-peak maximum slope, where hepatic artery contribution is maximum (assuming portal venous contrast inflow and hepatic venous contrast outflow are negligible) and the post-splenic-peak maximum slope, where portal venous contribution is maximum (assuming arterial contrast inflow and hepatic venous contrast outflow reached a plateau and portal venous contribution to the hepatic vein contrast outflow is negligible). The time at which the maximum slope of the portal venous tissue time-enhance- 
ment-curve is reached often parallels a post-peak negative slope of the arterial time-enhancement curve. Although this phenomenon may systematically lead to some underestimation of the portal venous liver-tissue perfusion, this is especially clear in regions where arterial perfusion is very high, such as rim regions surrounding liver tumors [20]. The increase in scan range allows us to scan entire volumes of the liver at the cost of a lower temporal resolution. This has several drawbacks, such as a decrease in signal-to-noise ratio and the possible scenario of missing an aortic or portal venous peak enhancement during the interscan interval, which may lead to an overestimation of the flow. However, scanning the entire liver volume solves the problem of having no visible portal vein present within the selected slice, which often was the case in single-level CTP.

Compared to contrast-enhanced CT, MRI and especially SPIO-enhanced MRI seem to have a slightly superior sensitivity and specificity whereas PET clearly has an inferior sensitivity but superior specificity for CRLM; furthermore PET can also detect more patients with extrahepatic disease [33]. The lower costs and widespread availability however may favor the use of CT over MRI despite the radiation dose, especially if the accuracy of detection and characterization of focal liver lesions further improves with dynamic or at least multiphase CT.

To conclude, total-liver-volume CTP is a noninvasive, well-tolerated, quantifiable and feasible but time-consuming technique, which can be implemented in routine preoperative and follow-up examinations of patients with liver metastases. The combination of automated and especially manual image fusion improves the quality of blood-flow maps and enables more reliable calculations of liver tissue perfusion, especially in subdiaphragmatic ROIs most vulnerable to distortion artifacts induced by differences in inspiratory volume. Preliminary results suggest an improved detection of colorectal liver metastases, although differentiation with benign focal perfusion alterations remains difficult and needs to be investigated in future work. Future software developments should focus on shortening reconstruction time and making nonlinear improved image registration possible.

Open Access This article is distributed under the terms of the Creative Commons Attribution Noncommercial License which permits any noncommercial use, distribution, and reproduction in any medium, provided the original author(s) and source are credited.

\section{References}

1. Abdalla EK, Vauthey JN, Ellis LM, Ellis V, Pollock R, Broglio KR, Hess K, Curley SA (2004) Recurrence and outcomes following hepatic resection, radiofrequency ablation, and combined resection/ablation for colorectal liver metastases. Ann Surg 239:818-825

2. Ruers TJ, de Jong KP, Ijzermans JN (2005) Radiofrequency for the treatment of liver tumours. Dig Surg 22:245-253

3. Kopp AF, Heuschmid M, Claussen CD (2002) Multidetector helical CT of the liver for tumour detection and characterization. Eur Radiol 12:745-752

4. Oliver JH III, Baron RL (1996) Helical biphasic contrast-enhanced CT of the liver: technique, indications, interpretation, and pitfalls. Radiology 201:1-14

5. Platt JF, Francis IR, Ellis JH, Reige KA (1997) Liver metastases: early detection based on abnormal contrast material enhancement at dual-phase helical CT. Radiology 205:49-53

6. Miles KA, Hayball M, Dixon AK (1991) Colour perfusion imaging: a new application of computed tomography. Lancet 337:643-645

7. Miles KA, Hayball MP, Dixon AK (1993) Functional images of hepatic perfusion obtained with dynamic CT. Radiology 188:405-411
8. Leggett DA, Kelley BB, Bunce IH, Miles KA (1997) Colorectal cancer: diagnostic potential of CT measurements of hepatic perfusion and implications for contrast enhancement protocols. Radiology 205:716-720

9. Platt JF, Francis IR, Ellis JH, Reige KA (1997) Difference in global hepatic enhancement assessed by dynamic CT in normal subjects and patients with hepatic metastases. J Comput Assist Tomogr 21:348-354

10. Miles KA, Leggett DA, Kelley BB, Hayball MP, Sinnatamby R, Bunce I (1998) In vivo assessment of neovascularisation of liver metastases using perfusion CT. Br J Radiol 71:276-281

11. Warren HW, Gallagher H, Hemingway DM, Angerson WJ, Bessent RG, Wotherspoon H, McArdle CS, Cooke TG (1998) Prospective assessment of the hepatic perfusion index in patients with colorectal cancer. Br J Surg 85:1708-1712

12. Dugdale PE, Miles KA (1999) Hepatic metastases: the value of quantitative assessment of contrast enhancement on computed tomography. Eur J Radiol 30:206-213

13. Cuenod C, Leconte I, Siauve N, Resten A, Dromain C, Poulet B, Frouin F, Clément O, Frija G (2001) Early changes in liver perfusion caused by occult metastases in rats: detection with quantitative CT. Radiology 218:556561
14. Tsushima Y, Blomley MJ, Yokoyama $\mathrm{H}$, Kusano S, Endo K (2001) Does the presence of distant and local malignancy alter parenchymal perfusion in apparently disease-free areas of the liver. Dig Dis Sci 46:2113-2119

15. Tsushima Y, Blomley MJ, Kusano S, Endo K (2002) Measuring portal venous perfusion with contrast-enhanced $\mathrm{CT}$ : comparison of direct and indirect methods. Acad Radiol 9:276-282

16. Tsushima Y, Funabasama S, Sanada S, Aoki J, Endo K (2002) Development of perfusion CT software for personal computers. Acad Radiol 9:922-926

17. Tsushima Y, Funabasama S, Aoki J, Sanada S, Endo K (2004) Quantitative perfusion map of malignant liver tumours, created from dynamic computed tomography data. Acad Radiol 11:215223

18. Miles KA, Colyvas K, Griffiths MR, Bunce IH (2004) Colon cancer: risk stratification using perfusion CT. Eur Radiol 14:129

19. Ganeshan B, Miles KA, Young RC, Chatwin CR (2007) Hepatic enhancement in colorectal cancer: texture analysis correlates with hepatic hemodynamics and patient survival. Acad Radiol 14:1520-1530 
20. Meijerink MR, van Cruijsen $H$, Hoekman K, Kater M, van Schaik C, van Waesberghe JH, Giaccone G, Manoliu RA (2007) The use of perfusion CT for the evaluation of therapy combining AZD2171 with gefitinib in cancer patients. Eur Radiol 17:17001713

21. Oken MM, Creech RH, Tormey DC, Horton J, Davis TE, McFadden ET, Carbone PP (1982) Toxicity and response criteria of the Eastern Cooperative Oncology Group. Am J Clin Oncol 5:649-655

22. Zankl M, Panzer W, Drexler G (1991) The calculation of dose from external photon exposures using reference humans and Monte Carlo methods IV. Organ doses from tomographic examinations. GSF report 30/91. GSF, Neuherberg, Germany

23. Zankl M, Panzer W, Drexler G (1993) Tomographic anthropomorphic models II. Organ doses from tomographic examinations. GSF report 30/93. GSF, Neuherberg, Germany
24. Stamm G, Nagel HD (2002) CT-expo: a novel program for dose evaluation in CT. Rofo 174:1570-1576

25. Miles KA (1991) Measurement of tissue perfusion by dynamic computed tomography. Br J Radiol 64:409-412

26. Bae KT, Heiken JP, Brink JA (1998) Aortic and hepatic contrast medium enhancement at CT. Part II. Effect of reduced cardiac output in a porcine model. Radiology 207:657-662

27. Miles KA, Kelley BB (1997) Altered perfusion adjacent to hepatic metastases. Clin Radiol 52:162-163

28. Ridge JA, Bading JR, Gelbard AS, Benua RS, Daly JM (1987) Perfusion of colorectal hepatic metastases. Relative distribution of flow from the hepatic artery and portal vein. Cancer 59:1547-1553

29. Kruskal JB, Thomas P, Kane RA, Goldberg SN (2004) Hepatic perfusion changes in mice livers with developing colorectal cancer metastases. Radiology 231:482-490

30. Irie T, Tsushima Y, Terahata S, Hatsuse K, Kusano S (1997) Rim enhancement in colorectal metastases at CT during infusion hepatic arteriography. Does it represent liver parenchyma or live tumour cell zone? Acta Radiol 38:416421
31. Terayama N, Matsui O, Ueda K, Kobayashi S, Sanada J, Gabata T, Kawamori Y, Kadoya M (2002) Peritumoral rim enhancement of liver metastasis: hemodynamics observed on single-level dynamic CT during hepatic arteriography and histopathologic correlation. J Comput Assist Tomogr 26:975-980

32. Semelka RC, Hussain SM, Marcos HB, Woosley JT (2000) Perilesional enhancement of hepatic metastases: correlation between MR imaging and histopathologic findings-initial observations. Radiology 215:89-94

33. Rappeport ED, Loft A, Berthelsen AK, von der Recke P, Larsen PN, Mogensen AM, Wettergren A, Rasmussen A, Hillingsoe J, Kirkegaard P, Thomsen C (2007) Contrast-enhanced FDG-PET/ $\mathrm{CT}$ vs. SPIO-enhanced MRI vs. FDGPET vs. CT in patients with liver metastases from colorectal cancer: a prospective study with intraoperative confirmation. Acta Radiol 48:369-378 